\begin{tabular}{|c|c|c|}
\hline Beitr. Ent. & Berlin & ISSN 0005-805X \\
\hline $49(1999) 2$ & S. $377-381$ & 13.09 .1999 \\
\hline
\end{tabular}

\title{
Due nuove Aleocharinae orofile e microttere della Cina ${ }^{1}$
}

\section{(Coleoptera: Staphylinidae)}

\author{
A 9 figuras
}

ROBERTO PACE

\section{Summary}

A new genus and two new species of Aleocharinae of the tribe Athetini are described and illustrated: Oroekklina g. n., daxuensis sp. n. from Sichuan, and Atheta (Microdota) puetzi from Shaanxi. The unusual new genus is briefly discussed.

\section{Zusammenfassung}

Eine neue Gattung und zwei neue Arten der Tribus Athetini (Aleocharinae) werden beschrieben und abgebildet: Oroekklina g. n., daxuensis sp. n. aus Sichuan sowie Atheta (Microdota) puetzi sp. n. aus Shaanxi. Die außergewöhnliche neue Gattung wird kurz erörtert.

\section{Key Words}

Coleoptera, Staphylinidae, Aleocharinae, Taxonomy, New genus, New species, China.

\section{Introduzione}

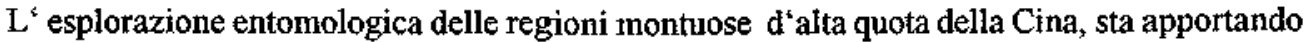
notevoli novità per quanto riguarda la conoscenza della sottofamiglia Aleocharinae. Un secondo esempio, dopo quello dato in precedenza per l'isola di Taiwan (PACE, 1995), è quello qui presentato, con la descrizione di un nuovo genere e di due nuove specie di Aleocharinae microttere affidatemi in studio da A. PUTz che le raccolse.

Gli holotypi sono conservati nelle collezioni del „Deutsches Entomologisches Institut" di Eberswalde (DEI).

${ }^{1}\left(154^{\circ}\right.$ Contributo alla conoscenza delle Aleocharinae) 


\section{Descrizioni}

Oroekklina gen. $\mathbf{n}$.

(Figg. 1-5)

Diagnosi. Habitus di Leptusa d'alta quota, formula tarsale 4-4-5, ma ligula di forma simile a quella del genere Atheta THOMSON. Anche il tipo di spermateca ha caratteri propri al genere Atheta e non ai generi della tribù Bolitocharini, come potrebbe indicare la formula tarsale.

Descrizione. Undici antennomeri; occhi molto ridotti; capo largo quanto il pronoto; tempie marginate all indietro; palpi labiali di 3 articoli, fig. 3; ligula a base stretta, divisa in due rami divergenti all'estremità distale, fig. 3; paraglosse non sporgenti in avanti, fig. 3; palpi mascellari di 4 articoli, fig. 5; lacinia con margine interno munito di una fila di spine apicale e di una spazzola setolosa preapicale, fig. 5 ; galea poco più lunga della lacinia, con corte setole apicali, fig. 5 ; mento trapezoidale, fig. 4 ; processo mesosternale acuto; mesocoxe contigue tra loro; formula tarsale 4-4-5; primo tarsomero posteriore corto; spermateca fig. 2.

Typus generis: Oroekklina daxuensis $\mathrm{sp} . \mathbf{n}$.

Etimologia: Il nome del nuovo genere significa "Colei che prende una svolta sulle montagne". Genere grammaticale femminile.

Discussione. La posizione sistematica del nuovo genere è problematica. In base alla presenza di 3 articoli dei palpi labiali e alla formula tarsale 4-4-5, il nuovo genere si collocherebbe nella tribù Bolitocharini, ma il tipo di spermateca e il tipo di ligula appartengono a generi della tribù Athetini. È la formula tarsale del nuovo genere che non consente una sicura attribuzione alla tribù Athetini (formula tarsale 4-5-5), perchè tutti i restanti caratteri generici del nuovo genere portano a attribuirlo senza difficoltà agli Athetini. Un caso simile si riscontra tra i generi Paraleptusa PEYERIMHOFF, 1901 e Geostiba THOMSON, 1858. Questi due generi mostrano ligula, palpi labiali e tipo di spermateca e di edeago affini. Solo Paraleptusa presenta formula tarsale 4-4-5 e Geostiba 4-5-5. Nonostante ciò Paraleptusa è sistematicamente da porre vicino a Geostiba. Lo stessa va fatto per il nuovo genere. Per il tipo di spermateca e per altri numerosi caratteri, il nuovo genere va collocato sistematicamente vicino al genere $A$ theta, sottogenere $M i c r o d o t a$, nonostante la presenza della formula tarsale 4-4-5.

Il nuovo genere è chiaramente differente dal genere Paraleptusa, diffuso nel bacino del Mediterraneo, sia per il tipo di spermateca, che per la forma della ligula (a base larga e a lati paralleli in Paraleptusa). Inoltre tra i due generi vi è differente ecologia: montano il nuovo genere, mediterraneo di bassa quota Paraleptusa.

\section{Oroekklina daxuensis gen. mov., sp. n.}

(Figg. 1-5)

Tipo. Holotypus o, China, Sichuan, Ganzi pref.: Daxue Shan, Pass $16 \mathrm{~km}$ W Kanding, 4290 m, 25. V.1997, A. Pütz leg. (DEI).

Descrizione. Lunghezza 1,8 mm. Corpo lucido e nero; antenne nere con antennomeri basali primo e secondo nero-bruni; zampe brune con femori rossicci. La microreticolazione della superficie del capo è svanita, quella del pronoto e delle elitre è distinta e quella dell' addome è netta. 


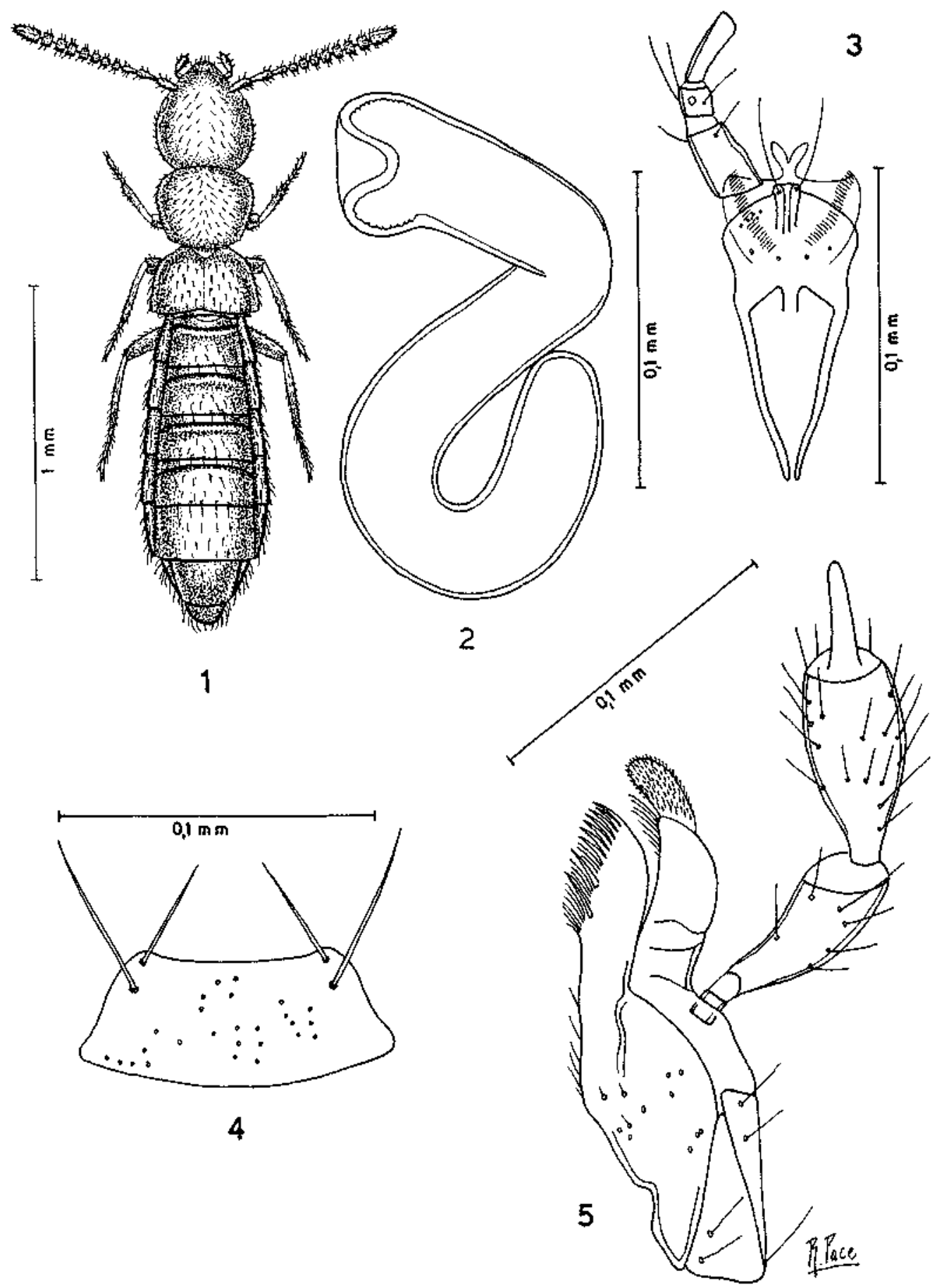

Figg. 1-5: Oroekklina daxuensis gen, n., sp. n., Sichuan: Daxie Shan. 1. habitus; 2. spermateca; 3. Labio con palpo labiale; 4. mento; 5. maxilla con palpo mascellare. 


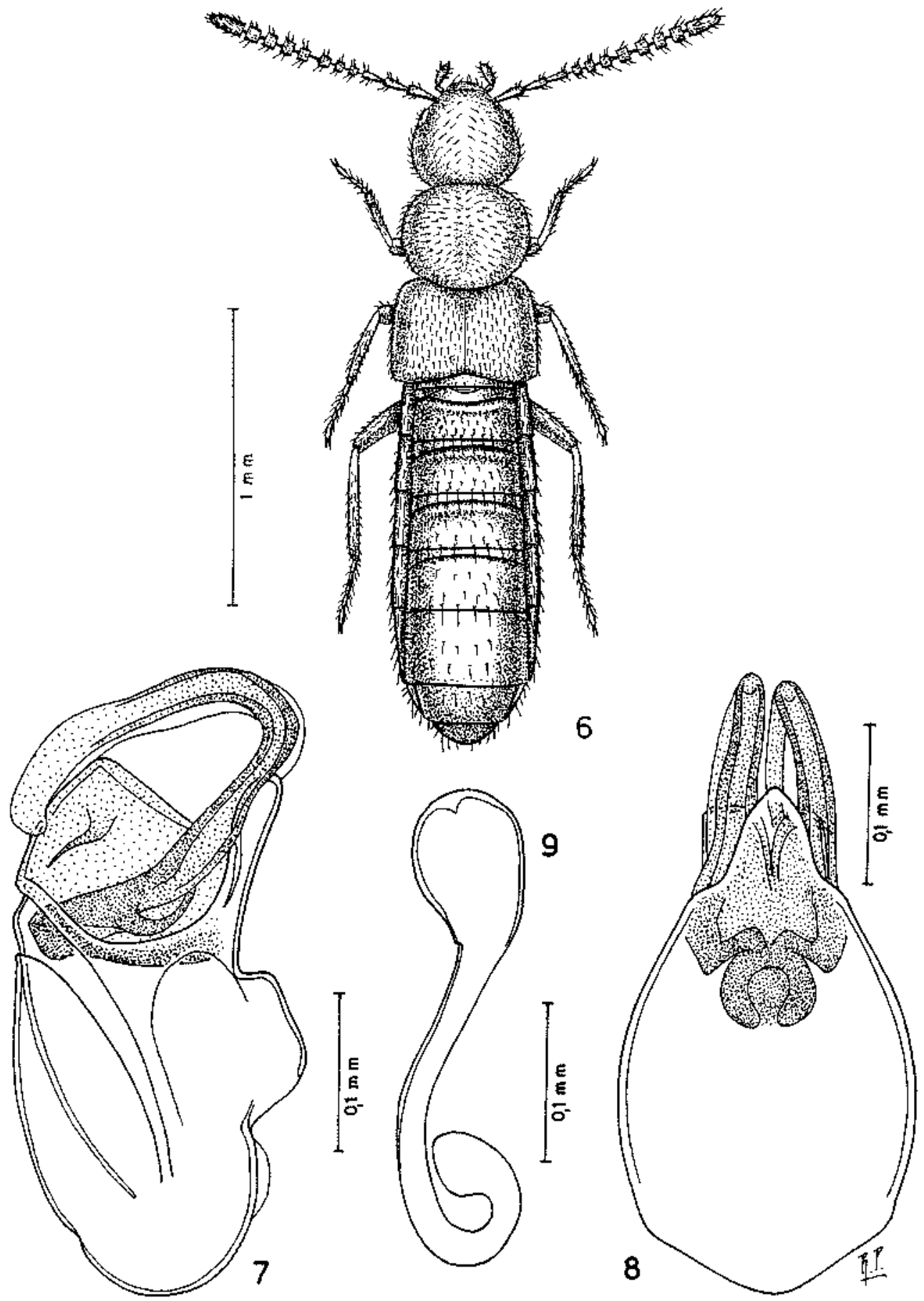

Figg. 6-8: Atheta (Microdota) puetzi sp. n., Shaanxi: Qinling Shan. 6. habitus; 7, edeago in visione laterale; 8 . lo stesso in visione laterale; 9 . spermateca. 
I granuli che coprono la superficie del capo sono poco distinti e sono assenti sulla fascia longitudinale mediana, quelli del pronto sono superficiali, quelli delle elitre sono salienti e quelli dell' addome sono ben distinti. L' addome presenta quattro solchi transversi basali degli uroterghi. Spermateca fig. 2.

\section{Atheta (Microdota) puetzi sp. n.} (Figg. 6-9)

Tipi. Holotypus $\sigma^{*}$, China, Shaanxi, Qinling Shan, Mountain W Pass at Autoroute $\mathrm{km} \mathrm{70,47}$ km S Xian, 2300-2500 m, sifted, 26-30.VIII. 1995, A. Pütz leg. (DEI). - Paratypus: 19, stessa provenienza.

Descrizione. Lunghezza $2,2 \mathrm{~mm}$. Avancorpo debolmente lucido, addome lucido. Corpo giallo-bruno con uriti liberi terzo, quarto e quinto di un bruno chiaro; antenne nero-bnune con i due antennomeri basali e la base del terzo gialli; zampe gialle. La microreticolazione della superficie del capo e del pronoto è netta, quella delle elitre è svanita e quella dell' addome è distinta. I microgranuli che coprono la superficie del capo sono poco evidenti, quelli del pronoto sono salienti e quelli delle elitre e dell' addome sono svaniti. Edeago figg. 7-8, spermateca fig. 9.

Comparazioni. Il tipo di spermateca ha forma caratteristica appartenente al sottogenere Microdota MULSANT \& REY, 1873.

Nell' ambito di questo sottogenere, nel Nepal è nota una specie, A. loebliella PACE, 1990, che presenta l' armatura interna dell' edeago robusta e in parte fuoriuscita dall' orifizio apicale, come nell' edeago della nuova specie. Tuttavia l' armatura genitale dell' edeago della nuova specie è enormemente sviluppata $e$ in più è forternente piegata su sè stessa, mentre in loebliella l' armatura genitale è appena ricurva. Inoltre te elitre di loebliella sono molto più lunghe del pronoto e gli occhi sono più lunghi delle tempie.

\section{Ringraziamenti}

Ringrazio il sig. A. Pürz per avermi affidato in esame le due specie qui descritte. Un cordiale ringraziamento rivolgo al Prof. Dr. H. H. DATHE, direttore del „Deutsches Entomologisches Institut" di Eberswalde che ha accettato di pubblicare il presente lavoro.

\section{Bibliografia}

Mulsant, M. \& REY, C. 1873: Description de divers Coléoptères Brévipennes nouveaux ou peu connus. - Opusc. Entom. 15: 147-189.

PACE, R. 1990: Aleocharinae nepalesi del Museo di Ginevra. Parte III. Revisione delle specie himalayane del sottogenere Microdota MULSANT \& REY. - Rev. suisse Zool. 97: 901-979.

PACE, R. 1995: Aleocharinae orofile e attere di Taiwan. - Boll. Soc, entom. italiana 127: 22-26.

PeyerimhofF, P. DE. 1901: In: PEYerIMHOFF \& SAINTE-CLARE-DEVILL: Coléoptères nouveaux ou peu connus trouvés dans les Alpes-Maritimes et les Basses-Alpes. - L'Abeille 30: 53-72.

Indirizzo dell' autore:

ROBERTO PACE

Via Vittorio Veneto, 13

37032 Monteforte d'Alpone (Verona)

Italia 